\title{
Central insulin action in energy and glucose homeostasis
}

\author{
Leona Plum, ${ }^{1,2}$ Bengt F. Belgardt, ${ }^{1}$ and Jens C. Brüning ${ }^{1}$
}

1Department of Mouse Genetics and Metabolism, Institute for Genetics, and Center of Molecular Medicine, University of Cologne, Cologne, Germany. ${ }^{2}$ Klinik II und Poliklinik für Innere Medizin der Universität zu Köln, Cologne, Germany.

\begin{abstract}
Insulin has pleiotropic biological effects in virtually all tissues. However, the relevance of insulin signaling in peripheral tissues has been studied far more extensively than its role in the brain. An evolving body of evidence indicates that in the brain, insulin is involved in multiple regulatory mechanisms including neuronal survival, learning, and memory, as well as in regulation of energy homeostasis and reproductive endocrinology. Here we review insulin's role as a central homeostatic signal with regard to energy and glucose homeostasis and discuss the mechanisms by which insulin communicates information about the body's energy status to the brain. Particular emphasis is placed on the controversial current debate about the similarities and differences between hypothalamic insulin and leptin signaling at the molecular level.
\end{abstract}

\section{History and background}

More than 150 years ago, the French physiologist Claude Bernard identified the liver as a reservoir for glucose. Remarkably, he also showed in rabbits that "piqûre" (pricking) of the floor of the fourth ventricle of the brain resulted in glucosuria (1) and concluded that the CNS plays an essential role in the control of peripheral blood glucose levels. More recently, insulin signaling in neuronal cells has been shown to regulate life span, growth, reproduction, and energy homeostasis in primitive organisms such as Caenorhabditis elegans and Drosophila melanogaster $(2,3)$. The insulin/IGF-1 signal transduction pathway shows similar characteristics in C. elegans, D. melanogaster, rodents, and humans, pointing to an evolutionarily conserved mechanism (4). Signaling of the insulin/IGF-1 receptor homolog dauer formation-2 (DAF-2) via the class IA PI3K (AGE-1) pathway in C. elegans has been shown to control development, reproductive function, and longevity in response to environmental stimuli such as energy supply, a mechanism involving the forkhead-O transcription factor (FOXO) homolog DAF-16 $(5,6)$. Likewise, the insulin receptor substrate (IRS) protein homolog CHICO in D. melanogaster regulates somatic growth, reproduction, and lipid metabolism (7).

It has been shown that overexpression of $D$. melanogaster insulinlike peptides (dILPs) in the nervous system of fasted larvae suppresses the hunger-driven ingestion of food and that upregulation of D. melanogaster p70/S6 kinase (dS6K) activity in dILP neurosecretory cells leads to a diminished hunger response in fasted larvae (8). Furthermore, ablation of dILP neurons in the Drosophila brain was found to result in prolonged lifespan, reduced fertility, increased fasting glucose levels, increased storage of lipids and carbohydrates, and reduced tolerance to heat and cold $(2,9)$, clearly assigning neurosecretory cells a pivotal importance in the regulation of lifespan and fuel metabolism in D. melanogaster.

Nonstandard abbreviations used: AgRP, agouti-related peptide; ARC, arcuate nucleus of the hypothalamus; BBB, blood-brain barrier; dILP, Drosophila melanogaster insulin-like peptide; FOXO, forkhead-O transcription factor; IR, insulin receptor; IRS, insulin receptor substrate; $\mathrm{K}_{\mathrm{ATP}}$ channel, ATP-sensitive potassium channel; $\alpha$-MSH, $\alpha$-melanocyte-stimulating hormone; NPY, neuropeptide Y; $\mathrm{PIP}_{3}$, phosphatidylinositol $_{3,4,5}$-trisphosphate; POMC, proopiomelanocortin; $\mathrm{VMH}$, ventromedial hypothalamic nucleus.

Conflict of interest: The authors have declared that no conflict of interest exists. Citation for this article: J. Clin. Invest. 116:1761-1766 (2006). doi:10.1172/JCI29063.
The finding that insulin, like the adipocyte-derived hormone leptin, affects both neuropeptide expression in the hypothalamus and food intake has recently led to the appreciation of the brain as an insulin target tissue with regard to energy homeostasis in mammals as well $(10,11)$. Generation of mice with brain-specific insulin receptor (IR) deficiency, which display obesity and impaired fertility, ultimately assigned brain IR signaling a critical role in the central regulation of fuel metabolism and reproduction (12). Peripherally circulating insulin crosses the blood-brain barrier (BBB) in proportion to serum insulin levels via a saturable mechanism $(13,14)$. IRs are widely distributed in the brain, with the highest concentrations in the olfactory bulb, hypothalamus, cerebral cortex, cerebellum, and hippocampus $(15,16)$. Moreover, downstream effectors of insulin such as IRS proteins and PI3K isoforms exhibit distinct expression patterns in the CNS partially overlapping with those of the IR (17). Hormone binding to the IR leads to rapid autophosphorylation of the receptor followed by tyrosine phosphorylation of IRS proteins, inducing the activation of downstream pathways such as MAPK and the PI3K cascade (18). Stimulation of the latter pathway negatively regulates the activity of FOXO by promoting its export from the nucleus upon phosphorylation by protein kinase B/AKT (19) (Figure 1).

\section{Regulation of neuropeptide expression}

In mammals, delivery of insulin to the brain causes anorexigenic (appetite-suppressing) effects, resulting in a reduction in body weight (20-22). Inhibition of insulin signaling in the brain produces opposite (orexigenic) effects $(12,23,24)$. IRs are particularly highly expressed in the arcuate nucleus of the hypothalamus (ARC) (16). Here, they are coexpressed with the anorexigenic neuropeptides proopiomelanocortin (POMC), the precursor of $\alpha$-melanocyte-stimulating hormone $(\alpha-\mathrm{MSH})$, and cocaine- and amphetamine-regulated transcript (CART), as well as with the orexigenic neuropeptide Y (NPY) and the agouti-related peptide (AgRP) (25). For a long time, changes in the expression of these hypothalamic neuropeptides have been regarded as the pivotal mechanism mediating insulin's anorexigenic effects in the CNS. Administration of insulin to the third ventricle of the brain decreases expression of the orexigenic NPY in the ARC $(10,26)$. Furthermore, icv delivered insulin increases expression of POMC (25). In particular, 


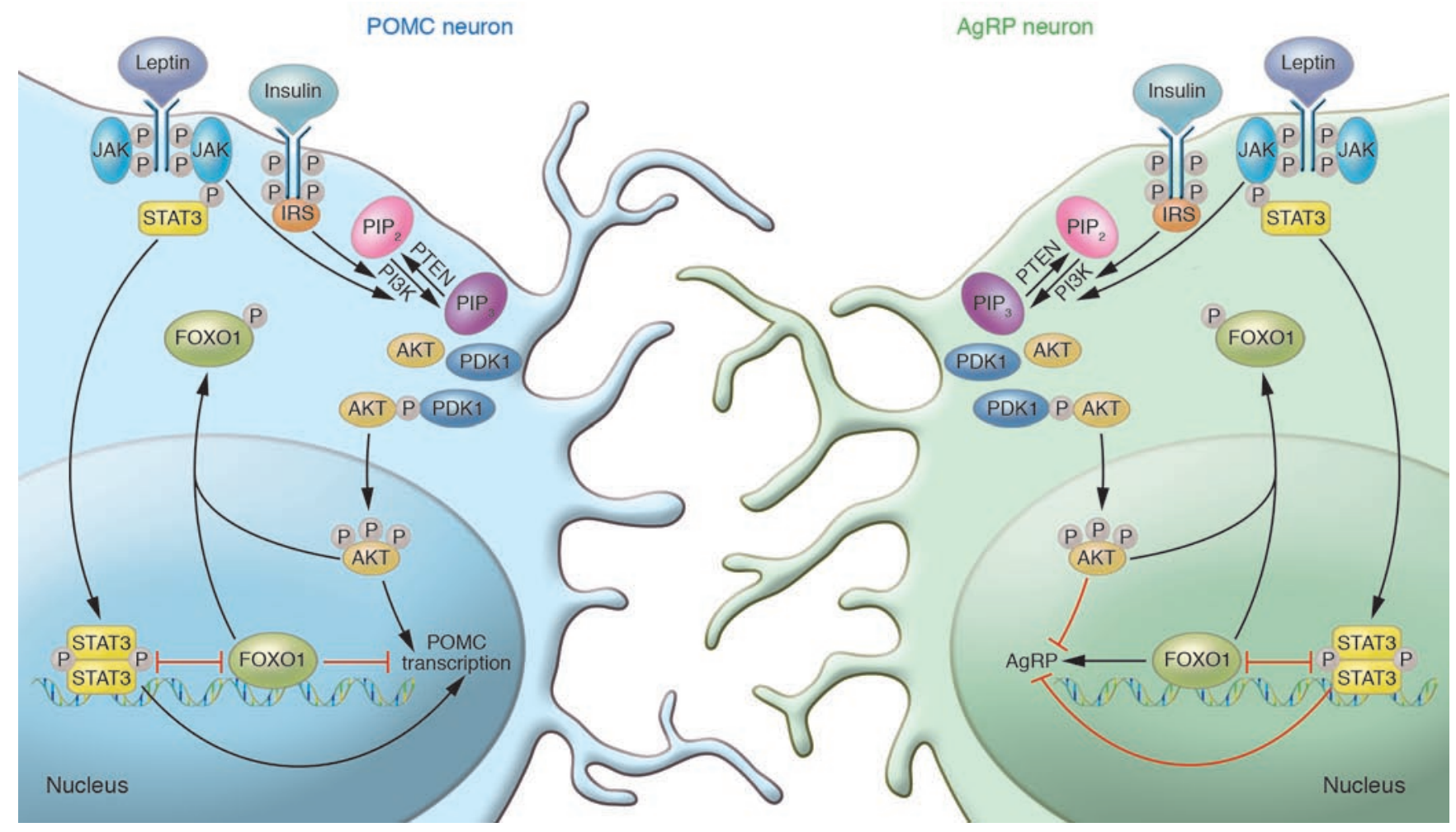

\section{Figure 1}

Insulin and leptin increase expression of POMC and decrease expression of AgRP. Insulin binds to its receptor on POMC and AgRP neurons, stimulating receptor autophosphorylation and activating its signal cascade. IRS proteins bind to the phosphorylated residues on the IR and subsequently recruit the regulatory subunit p85 of PI3K. PI3K thereby becomes activated and phosphorylates phosphatidylinositol-4,5-bisphosphate $\left(\mathrm{PIP}_{2}\right)$ on position $3^{\prime}$ in the inositol ring, generating $\mathrm{PIP}_{3}$. The lipid phosphatase PTEN antagonizes this by dephosphorylating PIP $\mathrm{P}_{3}$ to generate $\mathrm{PIP}_{2}$. The protein kinase B/AKT and phosphoinositide-dependent protein kinase 1 (PDK1) bind to PIP 3 via their pleckstrin homology $(\mathrm{PH})$ domains. AKT is readily phosphorylated and activated by PDK1. Phosphorylated AKT enters the nucleus, where it phosphorylates FOXO1. This leads to exclusion from the nucleus and thereby to inactivation of FOXO1. FOXO1 exerts different effects in POMC and AgRP neurons. In POMC neurons, it diminishes POMC transcription by recruiting Ncor and Hdac1 and by competing with binding sites for phosphorylated STAT3 in the promoter. By phosphorylating and excluding FOXO1 from the nucleus, insulin de-inhibits the promoter, thereby increasing POMC expression. In AgRP neurons, FOXO1 increases AgRP transcription. Here insulin decreases FOXO1-mediated transcription of AgRP by excluding FOXO1 from the nucleus. Leptin binds to its receptor, leading to recruitment of JAKs, which phosphorylate the receptor. The STAT3 monomer binds to the activated leptin receptor and is phosphorylated by JAKs. Upon phosphorylation, two STATs homodimerize and translocate to the nucleus, where they activate POMC transcription in POMC neurons and decrease AgRP transcription in AgRP neurons. It is possible that leptin also induces activation of PI3K. Consequently, insulin de-inhibits and leptin activates the POMC promoter. Insulin deactivates and leptin inhibits the AgRP promoter.

upregulation of $\alpha$-MSH was thought to mediate anorexigenic effects of insulin, as application of a melanocortin antagonist prevents the insulin-induced reduction in food intake (25). The exact interplay of these regulatory networks and their regulation by insulin has been the subject of numerous reviews (27-31). The analogous nature of insulin signaling to the well-characterized hypothalamic effects of leptin, which also suppresses NPY/AgRP expression and stimulates POMC expression, has led to the notion that insulin and leptin exert similar effects on regulatory entities in the hypothalamus.

Insulin and leptin activate signaling via different classes of receptor molecules: leptin signals through a cytokine receptor, and insulin signals through a membrane-bound tyrosine kinase receptor. Leptin robustly activates Pomc gene expression via activation of STAT3, while insulin fails to significantly activate STAT3 phosphorylation (32). However, recently a novel mechanism of PI3K/ FOXO1-dependent regulation of both AgRP and POMC tran- scription has been described. STAT3 and FOXO1 bind to partially overlapping motifs in the promoter region of both the Agrp and the Pomc genes (33). While STAT3 increases and FOXO1 decreases Pomc promoter activity, STAT3 activation decreases and FOXO1 increases Agrp promoter activity. Taking into account that leptin activates STAT3 and insulin inactivates FOXO1, transcriptional regulation of POMC and AgRP expression by these transcription factors may represent a mechanism to reconcile leptin and insulin signaling in the hypothalamus (Figure 1).

Although many studies have focused on the regulation of NPY/ AgRP transcription, the obvious lack of a phenotype in energy homeostasis of mice with targeted disruption of the Agrp and/or Npy gene(s) has put the importance of this pathway into question (34). This finding challenged the critical role of AgRP neurons in energy homeostasis. Only recently has a study demonstrated that AgRP-deficient mice exhibit an age-dependent lean phenotype arising from increased energy expenditure (35). Furthermore, 
two recent studies demonstrated that rapid and acute ablation of AgRP neurons in adult mice leads to dramatic anorexia $(36,37)$. In those studies, the diphtheria toxin receptor was expressed specifically in AgRP neurons. Thus, injection of diphtheria toxin, which is harmless to wild-type mouse cells, led to specific and rapid death of AgRP neurons. These findings show that the adult brain cannot compensate for the loss of AgRP neurons, yet the neonatal brain can, as ablation of the AgRP neurons in neonatal mice does not induce anorexia (37). The mechanisms compensating for the lack of AgRP neurons in neonatal mice but not in adult mice remain unknown. On the other hand, acute ablation of POMC neurons in adult mice leads to moderate-onset, mild hyperphagia, but those animals suffer from low levels of corticosterone due to ablation of POMC-expressing neurons in the pituitary; therefore, the full effect of acute POMC neuron ablation may be masked in those animals (36). Nevertheless, these studies underline the central importance of regulating AgRP/NPY and POMC neurons in adult mice to control energy homeostasis.

\section{PI3K as a unifying pathway for hypothalamic action of insulin and leptin}

A recent study has identified the PI3K signaling cascade as a common pathway activated by insulin as well as by leptin (38). Blockade of PI3K activation by icv administration of LY294002 inhibits both insulin's and leptin's acute anorexigenic effect in rats, assigning the PI3K pathway a key role in mediating the impact of both hormones on food intake $(39,40)$. However, what has received little attention is the exact site, time course, and magnitude of $\mathrm{PI} 3 \mathrm{~K}$ activation upon insulin and leptin stimulation. icv injection of leptin (as compared with insulin) produces only a transitory phosphorylation of IRS proteins and association of IRS with PI3K, but does not result in activation of AKT in whole hypothalamic extracts (32). In contrast to this transitory effect of leptin on IRS phosphorylation, leptin has been shown to robustly activate the MAPK pathway under the same experimental conditions (32). Thus, even though insulin and leptin signaling converge at the level of PI3K, the two hormones appear to elicit distinct signaling events downstream of PI3K.

Activation of PI3K by leptin and insulin has been demonstrated to differ depending on the cell type: while insulin and leptin act in parallel to stimulate PI3K in POMC neurons, they show opposing effects in AgRP neurons (41). Current research has increasingly focused on the differential aspect of insulin and leptin signaling at the level of single cells or homogenous cell populations (41-45). Transcriptional control of neuropeptide expression represents an important prerequisite for adequate functioning of anorexigenic and orexigenic neuronal circuits, but synaptic release of neuropeptide vesicles determines the physiological output of these cells. Thus, regulation of neuronal electrical activity represents a newly evolving focus of the field.

\section{Electrophysiological alterations in hypothalamic neurons}

Activation of PI3K, besides regulating neuropeptide expression via FOXO1, has been shown to regulate cell excitability via stimulation of ATP-sensitive potassium $\left(\mathrm{K}_{\mathrm{ATP}}\right)$ channels in the brain. Several mechanisms have been postulated for $\mathrm{K}_{\mathrm{ATP}}$ channel modulation by PI3K (Figure 2). It has been demonstrated that in addition to adenosine nucleotides, membrane phospholipids such as phosphatidylinositol ${ }_{4,5}$-bisphosphate $\left(\mathrm{PIP}_{2}\right)$ and phosphatidylinositol ${ }_{3,4,5}$-trisphosphate $\left(\mathrm{PIP}_{3}\right)$ bind to $\mathrm{K}_{\mathrm{ATP}}$ channels (46). Two mechanisms for the direct modulation of $\mathrm{K}_{\mathrm{ATP}}$ channel activity by $\mathrm{PIP}_{3}$ have been proposed: (a) $\mathrm{PIP}_{3}$ increases the probability that $\mathrm{K}_{\mathrm{ATP}}$ channels are open, which indirectly lowers the ability of ATP to inhibit the channels; and (b) $\mathrm{PIP}_{3}$ directly decreases ATP binding to the channel (46-48). Another indirect mechanism suggested for $\mathrm{PIP}_{3}$-dependent regulation of $\mathrm{K}_{\mathrm{ATP}}$ channels is local actin filament degradation around $\mathrm{K}_{\mathrm{ATP}}$ channels (49). The proteins that control and induce actin cytoskeletal degradation after $\mathrm{PIP}_{3}$ accumulation have not been identified yet, although Rho or Rac GTPases are likely candidates (50).

Spanswick et al. showed that $\mathrm{K}_{\mathrm{ATP}}$ channels, which are widely expressed in the hypothalamus (51), may function as the molecular end point of the pathway following leptin and IR activation in (unidentified) hypothalamic neurons $(52,53)$. This study suggested that the two hormones function in the hypothalamic control of energy homeostasis by altering electrical activity of their target cells. However, more detailed analyses complicate this view because (a) only $45 \%$ of unidentified ARC neurons follow this pattern (49); (b) activation of hypothalamic $\mathrm{K}_{\mathrm{ATP}}$ channels by insulin is abolished in obese rats (52); and (c) in identified POMC neurons, leptin stimulates while insulin inhibits electrical activity $(44,54)$, even though both hormones appear to stimulate PI3K in this cell type. This again points to discrete signaling pathways depending on the cell type and also on the metabolic situation.

Constitutive activation of $\mathrm{PIP}_{3}$ formation in POMC cells by conditional ablation of the phospholipid phosphatase and tensin homolog (PTEN) results in hyperphagia, diet sensitive obesity, and leptin resistance (45). This appears to result from augmented $\mathrm{PIP}_{3}$ dependent $K_{A T P}$ channel activation and consecutive electrical silencing of the POMC neurons. Similarly, insulin robustly activates $\mathrm{PIP}_{3}$ formation and leads to POMC cell hyperpolarization (44, 45). Insulin thereby can counteract leptin-evoked stimulation of POMC cell depolarization (45). These data clearly argue for differential, even opposing effects of insulin and leptin with respect to the regulation of POMC cell excitability.

Given that $\mathrm{K}_{\mathrm{ATP}}$ channels are expressed in POMC and NPY/AgRP neurons $(55,56)$, the aforementioned concept would suggest that insulin might electrically inactivate both types of neurons via PI3K-mediated $\mathrm{K}_{\mathrm{ATP}}$ activation, but this remains to be demonstrated for NPY neurons. In 2003, Schwartz et al. proposed a model in which physiological concentrations of leptin and insulin are catabolic effectors. Thus, anabolic stimuli would need to be powerful in order to overrule a physiologically catabolic basal state (57). It is tempting to speculate that insulin may inactivate not only anorexigenic POMC but also the more potent orexigenic NPY/AgRP cells, producing a net catabolic response. According to this model, insulin does not act analogously to leptin with respect to the regulation of POMC cell excitability. This notion is further supported by the different phenotypes of brain-specific IR versus brain-specific leptin receptor deficiency: while mice with brainspecific IR knockout show only a subtle, diet-dependent increase in body weight, brain leptin receptor deficiency appears to account for the severe obesity observed in $d b / d b$ mice $(12,58)$. Therefore, it will be important to determine the physiological role of differential effects of insulin and leptin signaling, such as regulation of POMC cell electrical activity, in the CNS.

\section{Effect of insulin on synaptic plasticity in the hypothalamus}

The ratio of excitatory to inhibitory synaptic input into a neuron determines not only direct cell-autonomous regulation of cell elec- 


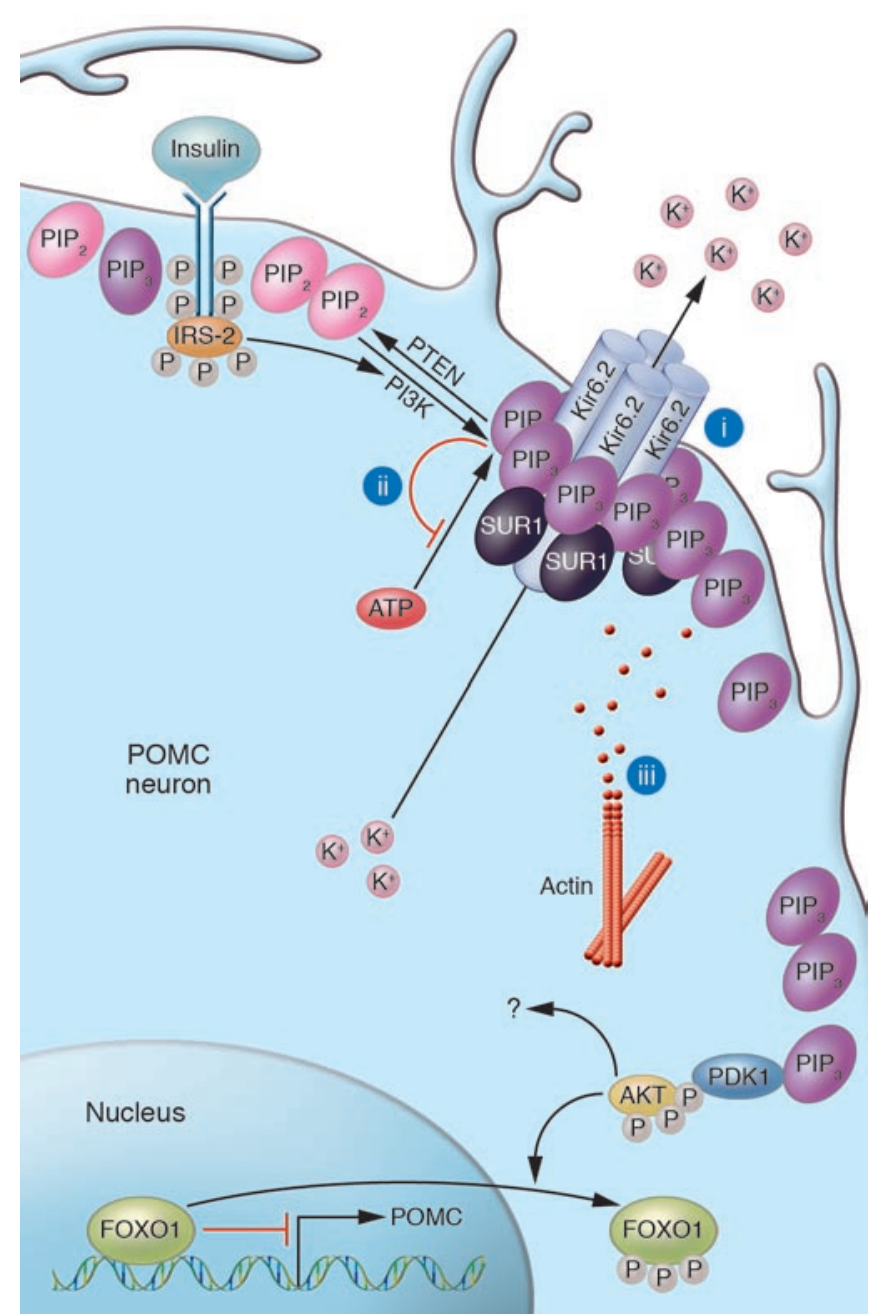

trical activity but also the set point of cell excitability. Recently, Pinto et al. introduced a novel concept regarding regulation of energy homeostasis by leptin-induced hypothalamic processes. They demonstrated that treating $o b / o b$ mice with leptin alters synaptic inputs to both NPY and POMC neurons. Upon leptin treatment, changes in synaptic contacts observed in $o b / o b$ mice reverted to the state observed in lean controls (59). However, it remains as yet undetermined whether these effects result from leptin action on postsynaptic (NPY and POMC) neurons, on the presynaptic projecting neurons, or on both. Emerging evidence has suggested that insulin signaling also plays a role in synaptic plasticity, as modulation of postsynaptic glutamate (excitatory) and GABA (inhibitory) receptor activities has been proposed to underlie this phenomenon in the hippocampus (60). However, the intracellular mechanisms involved in this process of synaptic rewiring have not been elucidated to date. This represents an exciting research focus for the future.

\section{Hypothalamic insulin action in glucose homeostasis}

Extraordinary progress in recent years has improved our understanding of the multiple roles of IR signaling in the hypothalamus. In addition to insulin's direct effects on the liver, its role in regulating hepatic glucose production (HGP) via signaling events

\section{Figure 2}

Generation of $\mathrm{PIP}_{3}$ leads to $\mathrm{K}_{\mathrm{ATP}}$ channel opening and consecutive cell hyperpolarization. Insulin activates PI3K, which phosphorylates $\mathrm{PIP}_{2}$ on position $3^{\prime}$ in the inositol ring, generating $\mathrm{PIP}_{3}$. The lipid phosphatase PTEN antagonizes this by dephosphorylating $\mathrm{PIP}_{3}$ to generate $\mathrm{PIP}_{2}$. $\mathrm{PIP}_{3}$ accumulation leads to activation of $\mathrm{K}_{\text {ATP }}$ channels and, thus, to potassium outflow. This leads to membrane hyperpolarization and silencing of the neuron. Three different mechanisms for channel opening have been suggested: (i) $\mathrm{PIP}_{3}$ binding to the Kir6.2 subunit of the potassium channel increases the probability that the channel is open, which indirectly lowers inhibition by ATP; (ii) $\mathrm{PIP}_{3}$ competes with ATP for binding to the Kir6.2 subunit, thereby lowering ATP's ability to close the channel; and (iii) $\mathrm{PIP}_{3}$ activates degradation of the local actin cytoskeleton. Also, activation of proteins downstream in the insulin cascade such as PDK1, AKT, glycogen synthase kinase 3 (GSK3), or mammalian target of rapamycin (mTOR) may be involved in insulin's ability to regulate $\mathrm{K}_{\text {ATP }}$ channel opening.

in the hypothalamus has drawn increasing attention. Obici et al. showed that icv injection of insulin or small-molecule insulin mimetics diminished hepatic glucose output. Interestingly, blockade of central $\mathrm{K}_{\mathrm{ATP}}$ channels with the sulfonylurea tolbutamide could abolish this effect (61). This work has led to the concept that besides regulating body weight homeostasis and reproductive endocrinology, hypothalamic insulin signaling also controls glucose utilization in the periphery, possibly via its action on central $\mathrm{K}_{\mathrm{ATP}}$ channels. Indeed, Pocai et al. recently showed that insulin acts on $\mathrm{K}_{\mathrm{ATP}}$ channels in hypothalamic neurons to control hepatic glucose production by decreasing glucose-6-phosphatase and phosphoenolpyruvate kinase expression in the liver (62). Transection of the vagal nerve to the liver nullified this effect, pointing to a role for the autonomous nervous system in transmitting insulin's central actions to the periphery. One recent publication implicates the cytokine IL- 6 as insulin's effector in the liver; it has been shown that insulin acting on unidentified neurons in the brain stimulates IL-6 secretion from as-of-now uncharacterized cells in the liver (63). Furthermore, IL-6 then activates STAT3 in hepatocytes and inhibits glucose production, and insulin's inhibiting effect on glucose production is blunted in animals lacking STAT3 in the liver (63). The relative importance of hypothalamic insulin signaling for the overall control of liver glucose production has lately been matter of debate, since another study demonstrated that a 4-fold rise in cranial insulin levels does not change acute hepatic glucose output in dogs (64). Thus, apparent differences between rodents and dogs concerning acute and chronic control of glucose homeostasis must be carefully addressed and assessed in future work.

Although the signaling events involved in $\mathrm{K}_{\mathrm{ATP}}$ channel activation have been extensively studied, the identity of the hypothalamic neurons that control hepatic glucose output has been elusive. The ARC has repeatedly been implicated as the crucial site for regulating peripheral glucose homeostasis. Using microinjection of adenovirus, Morton et al. demonstrated that expression of leptin receptor or a constitutively active mutant of AKT in the mediobasal hypothalamus led to improved peripheral insulin sensitivity and glucose response in rats lacking a functional leptin receptor. The insulin-sensitizing effect was partially independent of food intake and body weight (65). Gelling et al. also used the adenoviral delivery method to overexpress IRS2 or AKT in the mediobasal hypothalamus in diabetic rats (66). In this system glycemic response to insulin was enhanced 2 -fold, again indicating that PI3K signal- 
ing in the mediobasal hypothalamus acutely regulates peripheral glucose homeostasis. It is clear that when adenoviruses are used, expression of the delivered gene is not restricted to one type of neuron, such as POMC neurons, but is likely induced in different types of neurons found in the target nucleus. These studies have elegantly narrowed down the region that is critical for the regulation of peripheral glucose homeostasis; however, the identity of the responsible neuronal populations still needs to be pinpointed.

Further to this goal, icv infusion of NPY during a hyperinsulinemic-euglycemic clamp has been shown to weaken the suppression of glucose production by insulin in rats (67). This study characterized NPY/AgRP neurons as potential mediators of insulin's ability to suppress HGP via the CNS. As another prime candidate for regulating glucose homeostasis via central mechanisms, a subset of neurons located in the mediobasal hypothalamus has been suggested: neurons that sense changes in glucose itself. Interspersed in the ARC and the ventromedial hypothalamic nucleus (VMH) as well as the lateral hypothalamus are glucose-responsive neurons $(68,69)$. Two different classes of glucose-responsive neurons can be distinguished: glucose-excited neurons, which fire more frequently when ambient glucose levels rise; and glucose-inhibited neurons, which react to rising levels of glucose with hyperpolarization $(70,71)$. Many mechanisms for sensing changes in glucose levels have been proposed: changes in ATP/ADP ratios and subsequent $\mathrm{K}_{\mathrm{ATP}}$ channel opening; lactate generation by astroglial cells; direct binding of glucose to "glucose receptors"; or parallel entry of glucose and cations (70, 71 ). Song et al. found presynaptically glucose-excited and glucose-inhibited neurons in the VMH controlled by unidentified neurons from outside the VMH. Moreover, they noted that these circuits malfunction in rats prone to obesity (72). Kang et al. reported the detection of IR and glucose transporter 4 (which is regulated by insulin) in $40-75 \%$ of glucose-responsive neurons in the rat VMH (73). Demonstrating the complexity of the interactions among neuropeptides, neurotransmitters, and classical hormones, Wang et al. reported glucose-excited neurons in the rat ARC that were inactivated by NPY, activated by $\alpha-M S H$, and regulated by insulin depending on glucose concentration (74). Receiving input from both POMC and NPY neurons and having access to information about insulin and glucose levels make those neurons, in theory, a good prospect to acutely regulate glucose homeostasis. However, the exact identity of neurons in the control of peripheral glucose homeostasis is not known to date. Cell type-specific ablation of IR signaling molecules might help to ultimately define the exact site and mechanism involved in the regulation of peripheral glucose production.

\section{Perspectives}

Taken together, recent experiments have defined insulin action in the CNS as a critical determinant of energy homeostasis and of peripheral glucose metabolism in rodents.

Early studies investigated changes in mRNA expression of hypothalamic neuropeptides. These led to the identification of subsets of neurons in the CNS as critical regulators of energy homeostasis. However, the understanding of how the respective regions of the brain function within a complex network is still incomplete. One important issue is to further clarify the effects of hormones on neuronal electrical activity, since this will ultimately determine neuropeptide and/or neurotransmitter release. Additional downstream effects of insulin on the secretory machinery that amplify or suppress the secretory response will have to be elucidated. Complicating matters, electric activity and secretion are not necessarily coupled. It has, for example, been shown that in pancreatic $\beta$ cells, cyclic AMP works at a late stage of exocytosis, thereby exerting major effects on vesicle release despite only minor effects on electrical activity (75, 76). This phenomenon has also been observed in a wide variety of other secretory cell types including neurons (77). In order to correctly assess the activity of a secretory neuron, not only the production of neuropeptide/transmitters and the electrical activity, but also the effective synaptic output needs to be monitored.

Intercell communications also represent an important part of a functional network. Thus, it is important to unravel the connections, both anatomical and functional, between the target cells of hormones such as insulin and leptin. The use of refined techniques to characterize the exact site and molecular basis of insulin's homeostatic effects in the brain may ultimately allow for the development of novel therapeutic approaches for the treatment of type 2 diabetes mellitus and obesity.

\section{Acknowledgments}

The authors wish to thank Frances M. Ashcroft for fruitful discussion of the manuscript, Eva Rother for critical reading of the manuscript, and Gisela Schmall for excellent secretarial assistance. This work was supported by grants from the Köln Fortune Program (132/2003, 159/2004 to L. Plum), the Bundesministerium für Bildung und Forschung (ZMMK, TV-2 to J.C. Brüning), the European Union (LSHM-CT-2003-503041 to J.C. Brüning), and the Thyssen-Stiftung (10.04.1.153 to J.C. Brüning).

Address correspondence to: Jens C. Brüning, Institute for Genetics, Department of Mouse Genetics and Metabolism, Zülpicher Straße 47, 50674 Köln, Germany. Phone: 49-221-470-2467; Fax: 49-221470-5185; E-mail: jens.bruening@uni-koeln.de.
1. Bernard, C. 1855. Leçons de physiologie expérimentale appliquée à la médecine faites au Collège de France. Baillère et Fils. Paris, France. 296-313.

2. Rulifson, E.J., Kim, S.K., and Nusse, R. 2002. Ablation of insulin-producing neurons in flies: growth and diabetic phenotypes. Science. 296:1118-1120.

3. Wolkow, C.A., Kimura, K.D., Lee, M.S., and Ruvkun, G. 2000. Regulation of C. elegans life-span by insulinlike signaling in the nervous system. Science. 290:147-150.

4. Cheng, C.L., Gao, T.Q., Wang, Z., and Li, D.D. 2005. Role of insulin/insulin-like growth factor 1 signaling pathway in longevity. World J. Gastroenterol. 11:1891-1895.

5. Tissenbaum, H.A., and Ruvkun, G. 1998. An insulin-like signaling pathway affects both longevity and reproduction in Caenorhabditis elegans. Genet- ics. 148:703-717.

6. Ogg, S., et al. 1997. The Fork head transcription factor DAF-16 transduces insulin-like metabolic and longevity signals in C. elegans. Nature. 389:994-999.

7. Bohni, R., et al. 1999. Autonomous control of cell and organ size by CHICO, a Drosophila homolog of vertebrate IRS1-4. Cell. 97:865-875.

8. Wu, Q., Zhang, Y., Xu, J., and Shen, P. 2005. Regulation of hunger-driven behaviors by neural ribosomal 56 kinase in Drosophila. Proc. Natl. Acad. Sci. U. S. A. 102:13289-13294.

9. Broughton, S.J., et al. 2005. Longer lifespan, altered metabolism, and stress resistance in Drosophila from ablation of cells making insulin-like ligands. Proc. Natl. Acad. Sci. U. S. A. 102:3105-3110.

10. Schwartz, M.W., et al. 1992. Inhibition of hypothalamic neuropeptide Y gene expression by insulin.
Endocrinology. 130:3608-3616.

11. Baskin, D.G., et al. 1999. Insulin and leptin: dual adiposity signals to the brain for the regulation of food intake and body weight. Brain Res. 848:114-123.

12. Bruning, J.C., et al. 2000. Role of brain insulin receptor in control of body weight and reproduction. Science. 289:2122-2125.

13. Margolis, R.U., and Altszuler, N. 1967. Insulin in the cerebrospinal fluid. Nature. 215:1375-1376.

14. Woods, S.C., and Porte, D., Jr. 1977. Relationship between plasma and cerebrospinal fluid insulin levels of dogs. Am. J. Physiol. 233:E331-E334.

15. Havrankova, J., Roth, J., and Brownstein, M. 1978. Insulin receptors are widely distributed in the central nervous system of the rat. Nature. 272:827-829.

16. Van Houten, M., Posner, B.I., Kopriwa, B.M., and Brawer, J.R. 1979. Insulin-binding sites in the rat 
brain: in vivo localization to the circumventricular organs by quantitative radioautography. Endocrinology. 105:666-673.

17. Horsch, D., and Kahn, C.R. 1999. Region-specific mRNA expression of phosphatidylinositol 3-kinase regulatory isoforms in the central nervous system of C57BL/6J mice. J. Comp. Neurol. 415:105-120.

18. White, M.F. 2003. Insulin signaling in health and disease. Science. 302:1710-1711.

19. Biggs, W.H., 3rd, Meisenhelder, J., Hunter, T., Cavenee, W.K., and Arden, K.C. 1999. Protein kinase B/ Akt-mediated phosphorylation promotes nuclear exclusion of the winged helix transcription factor FKHR1. Proc. Natl. Acad. Sci. U. S. A. 96:7421-7426.

20. Woods, S.C., Lotter, E.C., McKay, L.D., and Porte, D., Jr. 1979. Chronic intracerebroventricular infusion of insulin reduces food intake and body weight of baboons. Nature. 282:503-505.

21. McGowan, M.K., Andrews, K.M., Fenner, D., and Grossman, S.P. 1993. Chronic intrahypothalamic insulin infusion in the rat: behavioral specificity. Physiol. Behav. 54:1031-1034.

22. Hallschmid, M., et al. 2004. Intranasal insulin reduces body fat in men but not in women. Diabetes. 53:3024-3029.

23. Air, E.L., et al. 2002. Small molecule insulin mimetics reduce food intake and body weight and prevent development of obesity. Nat. Med. 8:179-183.

24. Strubbe, J.H., and Mein, C.G. 1977. Increased feeding in response to bilateral injection of insulin antibodies in the VMH. Physiol. Behav. 19:309-313.

25. Benoit, S.C., et al. 2002. The catabolic action of insulin in the brain is mediated by melanocortins. J. Neurosci. 22:9048-9052.

26. Sipols, A.J., Baskin, D.G., and Schwartz, M.W. 1995. Effect of intracerebroventricular insulin infusion on diabetic hyperphagia and hypothalamic neuropeptide gene expression. Diabetes. 44:147-151.

27. Gerozissis, K. 2003. Brain insulin: regulation, mechanisms of action and functions. Cell. Mol. Neurobiol. 23:1-25.

28. Benoit, S.C., Clegg, D.J., Seeley, R.J., and Woods, S.C. 2004. Insulin and leptin as adiposity signals. Recent Prog. Horm. Res. 59:267-285.

29. Niswender, K.D., and Schwartz, M.W. 2003. Insulin and leptin revisited: adiposity signals with overlapping physiological and intracellular signaling capabilities. Front. Neuroendocrinol. 24:1-10.

30. Obici, S., and Rossetti, L. 2003. Minireview: nutrient sensing and the regulation of insulin action and energy balance. Endocrinology. 144:5172-5178.

31. Plum, L., Schubert, M., and Bruning, J.C. 2005. The role of insulin receptor signaling in the brain. Trends Endocrinol. Metab. 16:59-65.

32. Carvalheira, J.B., et al. 2005. Cross-talk between the insulin and leptin signaling systems in rat hypothalamus. Obes. Res. 13:48-57.

33. Kitamura, T., et al. 2006. Forkhead protein FoxO1 mediates Agrp-dependent effects of leptin on food intake. Nat. Med. 12:534-540.

34. Qian, S., et al. 2002. Neither agouti-related protein nor neuropeptide $\mathrm{Y}$ is critically required for the regulation of energy homeostasis in mice. $\mathrm{Mol}$. Cell. Biol. 22:5027-5035.

35. Wortley, K.E., et al. 2005. Agouti-related proteindeficient mice display an age-related lean phenotype. Cell Metab. 2:421-427.

36. Gropp, E., et al. 2005. Agouti-related peptideexpressing neurons are mandatory for feeding. Nat. Neurosci. 8:1289-1291.

37. Luquet, S., Perez, F.A., Hnasko, T.S., and Palmiter, R.D. 2005. NPY/AgRP neurons are essential for feeding in adult mice but can be ablated in neonates. Science. 310:683-685.
38. Niswender, K.D., et al. 2003. Immunocytochemical detection of phosphatidylinositol 3-kinase activation by insulin and leptin. J. Histochem. Cytochem. 51:275-283.

39. Niswender, K.D., et al. 2003. Insulin activation of phosphatidylinositol 3-kinase in the hypothalamic arcuate nucleus: a key mediator of insulin-induced anorexia. Diabetes. 52:227-231.

40. Niswender, K.D., et al. 2001. Intracellular signalling. Key enzyme in leptin-induced anorexia. Nature. 413:794-795.

41. Xu, A.W., et al. 2005. PI3K integrates the action of insulin and leptin on hypothalamic neurons. J. Clin. Invest. 115:951-958. doi:10.1172/JCI200524301.

42. Dhillon, H., et al. 2006. Leptin directly activates SF1 neurons in the VMH, and this action by leptin is required for normal body-weight homeostasis. Neuron. 49:191-203.

43. Balthasar, N., et al. 2004. Leptin receptor signaling in POMC neurons is required for normal body weight homeostasis. Neuron. 42:983-991.

44. Choudhury, A.I., et al. 2005. The role of insulin receptor substrate 2 in hypothalamic and $\beta$ cell function. J. Clin. Invest. 115:940-950. doi:10.1172/ JCI200524445.

45. Plum, L., et al. 2006. Enhanced $\mathrm{PIP}_{3}$ signaling in POMC neurons causes $\mathrm{K}_{\mathrm{ATP}}$ channel activation and leads to diet-sensitive obesity. J. Clin. Invest. 116:1886-1901. doi:10.1172/JCI27123.

46. Shyng, S.L., and Nichols, C.G. 1998. Membrane phospholipid control of nucleotide sensitivity of KATP channels. Science. 282:1138-1141.

47. Baukrowitz, T., et al. 1998. PIP2 and PIP as determinants for ATP inhibition of KATP channels. Science. 282:1141-1144.

48. MacGregor, G.G., et al. 2002. Nucleotides and phospholipids compete for binding to the $\mathrm{C}$ terminus of KATP channels. Proc. Natl. Acad. Sci. U. S. A 99:2726-2731.

49. Mirshamsi, S., et al. 2004. Leptin and insulin stimulation of signalling pathways in arcuate nucleus neurones: PI3K dependent actin reorganization and KATP channel activation. BMC Neurosci. 5:54.

50. Ridley, A.J., and Hall, A. 1992. Distinct patterns of actin organization regulated by the small GTPbinding proteins Rac and Rho. Cold Spring Harb. Symp. Ouant. Biol. 57:661-671.

51. Karschin, C., Ecke, C., Ashcroft, F.M., and Karschin, A. 1997. Overlapping distribution of K(ATP) channel-forming Kir6.2 subunit and the sulfonylurea receptor SUR1 in rodent brain. FEBS Lett. 401:59-64.

52. Spanswick, D., Smith, M.A., Mirshamsi, S., Routh, V.H., and Ashford, M.L. 2000. Insulin activates ATPsensitive $\mathrm{K}+$ channels in hypothalamic neurons of lean, but not obese rats. Nat. Neurosci. 3:757-758.

53. Spanswick, D., Smith, M.A., Groppi, V.E., Logan, S.D., and Ashford, M.L. 1997. Leptin inhibits hypothalamic neurons by activation of ATP-sensitive potassium channels. Nature. 390:521-525.

54. Cowley, M.A., et al. 2001. Leptin activates anorexigenic POMC neurons through a neural network in the arcuate nucleus. Nature. 411:480-484.

55. Dunn-Meynell, A.A., Rawson, N.E., and Levin, B.E. 1998. Distribution and phenotype of neurons containing the ATP-sensitive $\mathrm{K}+$ channel in rat brain. Brain Res. 814:41-54.

56. Ibrahim, N., et al. 2003. Hypothalamic proopiomelanocortin neurons are glucose responsive and express K(ATP) channels. Endocrinology. 144:1331-1340.

57. Schwartz, M.W., et al. 2003. Is the energy homeostasis system inherently biased toward weight gain? Diabetes. 52:232-238.

58. De Luca, C., et al. 2005. Complete rescue of obe- sity, diabetes, and infertility in $\mathrm{db} / \mathrm{db}$ mice by neuron-specific LEPR-B transgenes. J. Clin. Invest. 115:3484-3493. doi:10.1172/JCI24059.

59. Pinto, S., et al. 2004. Rapid rewiring of arcuate nucleus feeding circuits by leptin. Science. 304:110-115.

60. Wan, Q., et al. 1997. Recruitment of functional GABA(A) receptors to postsynaptic domains by insulin. Nature. 388:686-690.

61. Obici, S., Zhang, B.B., Karkanias, G., and Rossetti, L. 2002. Hypothalamic insulin signaling is required for inhibition of glucose production. Nat. Med. 8:1376-1382.

62. Pocai, A., et al. 2005. Hypothalamic K(ATP) channels control hepatic glucose production. Nature. 434:1026-1031.

63. Inoue, H., et al. 2006. Role of hepatic STAT3 in brain-insulin action on hepatic glucose production. Cell Metab. 3:267-275.

64. Edgerton, D.S., et al. 2006. Insulin's direct effects on the liver dominate the control of hepatic glucose production. J. Clin. Invest. 116:521-527. doi:10.1172/JCI27073.

65. Morton, G.J., et al. 2005. Leptin regulates insulin sensitivity via phosphatidylinositol-3-OH kinase signaling in mediobasal hypothalamic neurons. Cell Metab. 2:411-420.

66. Gelling, R.W., et al. 2006. Insulin action in the brain contributes to glucose lowering during insulin treatment of diabetes. Cell Metab. 3:67-73.

67. Van den Hoek, A.M., et al. 2004. Intracerebroventricular neuropeptide Y infusion precludes inhibition of glucose and VLDL production by insulin. Diabetes. 53:2529-2534.

68. Oomura, Y., Ono, T., Ooyama, H., and Wayner, M.J. 1969. Glucose and osmosensitive neurones of the rat hypothalamus. Nature. 222:282-284.

69. Anand, B.K., Chhina, G.S., Sharma, K.N., Dua, S., and Singh, B. 1964. Activity of single neurons in the hypothalamic feeding centers: effect of glucose. Am. J. Physiol. 207:1146-1154.

70. Levin, B.E., Routh, V.H., Kang, L., Sanders, N.M., and Dunn-Meynell, A.A. 2004. Neuronal glucosensing: what do we know after 50 years? Diabetes. 53:2521-2528.

71. Burdakov, D., Luckman, S.M., and Verkhratsky, A. 2005. Glucose-sensing neurons of the hypothalamus. Philos. Trans. R. Soc. Lond. B Biol. Sci. 360:2227-2235.

72. Song, Z., Levin, B.E., McArdle, J.J., Bakhos, N., and Routh, V.H. 2001. Convergence of pre- and postsynaptic influences on glucosensing neurons in the ventromedial hypothalamic nucleus. Diabetes. 50:2673-2681.

73. Kang, L., Routh, V.H., Kuzhikandathil, E.V., Gaspers, L.D., and Levin, B.E. 2004. Physiological and molecular characteristics of rat hypothalamic ventromedial nucleus glucosensing neurons. Diabetes. 53:549-559.

74. Wang, R., et al. 2004. The regulation of glucoseexcited neurons in the hypothalamic arcuate nucleus by glucose and feeding-relevant peptides. Diabetes. 53:1959-1965.

75. Barnett, D.W., Pressel, D.M., Chern, H.T., Scharp, D.W., and Misler, S. 1994. cAMP-enhancing agents "permit" stimulus-secretion coupling in canine pancreatic islet beta-cells. J. Membr. Biol. 138:113-120.

76. Hashiguchi, H., et al. 2006. Cyclic AMP/cAMP-GEF pathway amplifies insulin exocytosis induced by $\mathrm{Ca} 2+$ and ATP in rat islet beta-cells. Diabetes Metab. Res. Rev. 22:64-71.

77. Evans, G.J., and Morgan, A. 2003. Regulation of the exocytotic machinery by cAMP-dependent protein kinase: implications for presynaptic plasticity. Biochem. Soc. Trans. 31:824-827. 